\title{
SIMPLE METHOD FOR GAIT ASSESENT
}

\author{
Abdullah Amin S. Abdullah¹, Alionte Cristian-Gabriel2, Ungureanu Liviu-Marian³, Comeaga Daniel-Constatin ${ }^{4}$ \\ 1,2,3,4 University Politehnica of Bucharest \\ Splaiul Indepenentei 313, sector 6, Bucuresti, Romania, RO-060042 \\ email: cristian.alionte@upb.ro
}

\begin{abstract}
This article presents a method used for investigating the kinematic parameters of the inferior limb at the knee and ankle articulations, while the patient is gait using a simple mechanical system. The method is a visual one and is based on the processing of captured images during gait that can be done manually or automatically. The measured parameters will be used during patient's locomotor recovery activities. It is known that, in locomotion it is essential to study the positions of the segments of the lower limb and the angles between them and the angular velocities and accelerations in the joints. The construction of the mechanical system envisaged the use of components having the lowest possible weight and low cost.
\end{abstract}

Keywords: virtual planar two-link manipulator, gait assessment.

\section{Introduction}

It is well known that any movement of segments of the locomotor apparatus is accomplished by nerve impulses emitted by the brain which are received by the muscles. However, in the event of paralysis, nerve impulses no longer transmit to the muscles due to deterioration or disruption of the nerve path, especially in lower spine injuries and this will induce disease as: tetraplegia, paraplegia, hemiplegia, etc. Under certain complex conditions of mechanical and informational stimulation, it is possible to restore the transmission of nerve impulses and thus to improve the degree of disability of the person, which contributes substantially to the improvement of his living conditions. These results can be obtained by stimulation of the paralyzed limbs and they are procedures that combine, in a rigorous synchronization, routine movements representing a process of reeducation of the person, movements that have been learned since childhood when it was valid, with the stimulation of specific nerves to obtain these movements, for example: gait in a straight line on a horizontal surface combined with simultaneous movement of the arms, skiing, etc.

These complex procedures are based on the realization of a mechatronic gait simulation system.

This system needs as input parameters those which are as closest possible with the real ones owned by the patient because these parameters are individualized for each individual due to various factors:

- Body features: height and weight;

- Anatomy of the person: length of body segments, muscle tone;
- Psychosomatic features: personality, activism, type of life (sedentary, sporting), etc.;

- How to get the go. During childhood, there is an individualized system for each person to acquire two-leg and gait body stability mechanisms.

- Gait mechanism is refreshed continuously. This takes place throughout the life span.

- Gait research is done by averaging and therefore it cannot be said that gait is correct unless it adversely affects other components of the human body including gait

Starting from these reasons, we can conclude that when it is desired to develop a medical rehabilitation system for walking, it is important to look at the individual kinetic parameters of the patient. This makes it important to implement a simpler walking analysis system that can be adapted to any patient and does not lead to a change in the usual course of the patient.

In literature can be found several studies regarding human gait. One interesting study [4] demonstrates that gait analysis can be used to analyze gait adaptations for patients with knee problems. One possible solution like ours [5] includes algorithms to determine event times for gait based solely on kinematic data. Finally, In the paper [6] is presented a device with a high degree of portability that can be used both laboratory and ambulatory. As a conclusion, the main issues which can be founded are that a special space for experiments is needed, the patient preparation take a long time and the devices are expansive. In developing of the method, we had as goal to solve these issues using a planar two-link manipulator mechanism. 
The system was developed within the framework of the dissertation thesis of $\mathrm{Mr}$. ABDULLAH Amin S. Abdullah, which aimed at researching the design and realization of a biomechanical system for the medical rehabilitation of the inferior limb by a continuous passive movement of the foot. Consideration has been given to balancing several factors in the design process, including cost and simplicity of design.

\section{Design Considerations}

All these considerations led to the realization of the planar two-linked system shown in Figure 1 which permits automatic processing of the gait pattern by monitoring and determining, with the help of a video camera, of the two angles of the knee and ankle between the corresponding segments. For this reason, the system was made of a material with a color (white) that contrasts with the laboratory environment.
The system has three segments: one corresponding to the virtual line between the calcaneus and the distal phalange III (fixed element 1), the second on the virtual line between the tibia and the peroneus (adjustable element 2) and the third at thigh zone parallel to the femur (adjustable element 3) between which there are two cylindrical joints: at the knee (4) and the ankle (5).

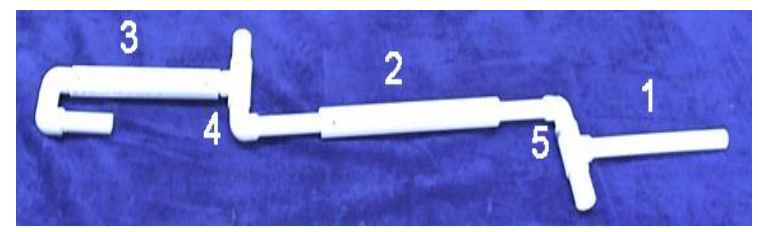

Figure 1: The planar two-linked system used to investigate the kinematic parameters of the gait:

1 - segment corresponding to the foot;

2 - segment corresponding to calf;

3 - segment corresponding to thigh;

4-cylindrical articulation of the knee,

5-cylindrical ankle joint

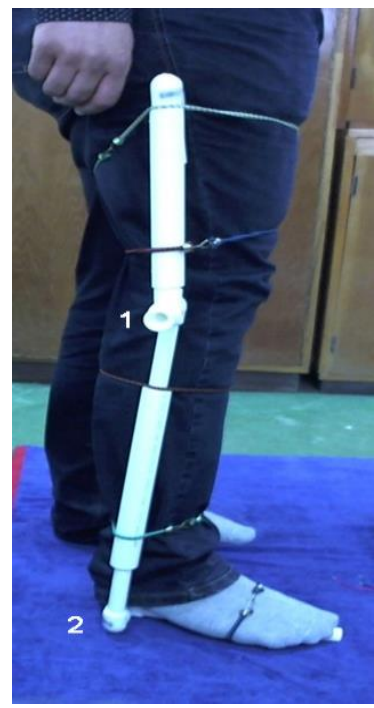

Figure 2: Hinging of the system on the patient:

1- cylindrical knee joint; 2 - ankle joint

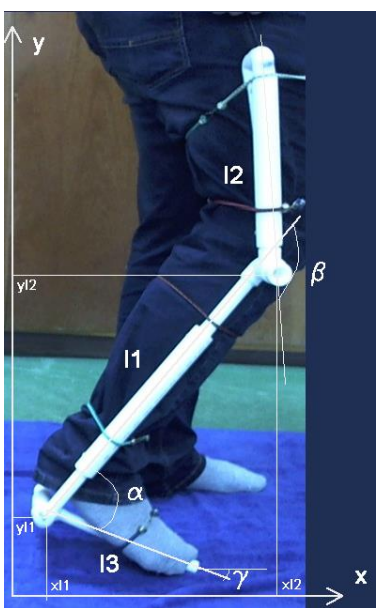

Figure 3: Base model used in parameters calculus 
The assembly is made up of two joints located at the knee (1) and ankle (2) respectively. The grip is made at the level of the thigh in two points, at the level of the calf in two points and at the foot at a single point as it can be seen in figure 2 . The system can vary the length of the segmented grip on the stem. The mechanical foot attachment is achieved by means of elastic strings placed at the level of the pulley and the calf at two points in each segment.

\section{Parameters Calculus}

If only one foot is considered and we do not consider the whole sequence of gait, the position and speed parameters for the ankle position at the lateral malleolus point $(\mathrm{xl} 1, \mathrm{yl} 1)$ can be determined based on the kinematic model of the structure (Figure 3) using equations:

$$
\left(\begin{array}{l}
x_{l 1} \\
y_{l 1}
\end{array}\right)=\left(\begin{array}{c}
l_{3} \cdot \cos \gamma+l_{1} \cdot \cos (\gamma+\alpha) \\
l_{3} \cdot \sin \gamma+l_{1} \cdot \sin (\gamma+\alpha)
\end{array}\right)
$$

We can rewrite the Jacobian equation for speed:

$$
\left(\begin{array}{l}
\dot{x}_{l 1} \\
\dot{y}_{l 1}
\end{array}\right)=\left[\begin{array}{cc}
-l_{3} \cdot \sin \gamma-l_{1} \cdot \sin (\gamma+\alpha) & -l_{1} \cdot \sin (\gamma+\alpha) \\
l_{3} \cdot \cos \gamma+l_{1} \cdot \cos (\gamma+\alpha) & l_{1} \cdot \cos (\gamma+\alpha)
\end{array}\right]\left[\begin{array}{c}
\dot{\gamma} \\
\dot{\alpha}
\end{array}\right]
$$

If only one leg is considered and we do not consider the entire sequence of motion, the kinematic model of the structure (Figure 3) can determine the position and speed parameters for the knee position (xl2, yl2) using the equation:

$$
\left(\begin{array}{l}
x_{l 2} \\
y_{l 2}
\end{array}\right)=\left(\begin{array}{c}
l_{1} \cdot \cos \alpha+l_{2} \cdot \cos (\alpha+\beta) \\
l_{1} \cdot \sin \alpha+l_{2} \cdot \sin (\alpha+\beta)
\end{array}\right)
$$

We can rewrite the Jacobian equation for speed:

$$
\left(\begin{array}{c}
\dot{x}_{l 2} \\
\dot{y}_{l 2}
\end{array}\right)=\left[\begin{array}{cc}
-l_{1} \cdot \sin \alpha-l_{2} \cdot \sin (\alpha+\beta) & -l_{2} \cdot \sin (\alpha+\beta) \\
l_{1} \cdot \cos \alpha+l_{2} \cdot \cos (\alpha+\beta) & l_{2} \cdot \cos (\alpha+\beta)
\end{array}\right]\left[\begin{array}{c}
\dot{\alpha} \\
\dot{\beta}
\end{array}\right]
$$

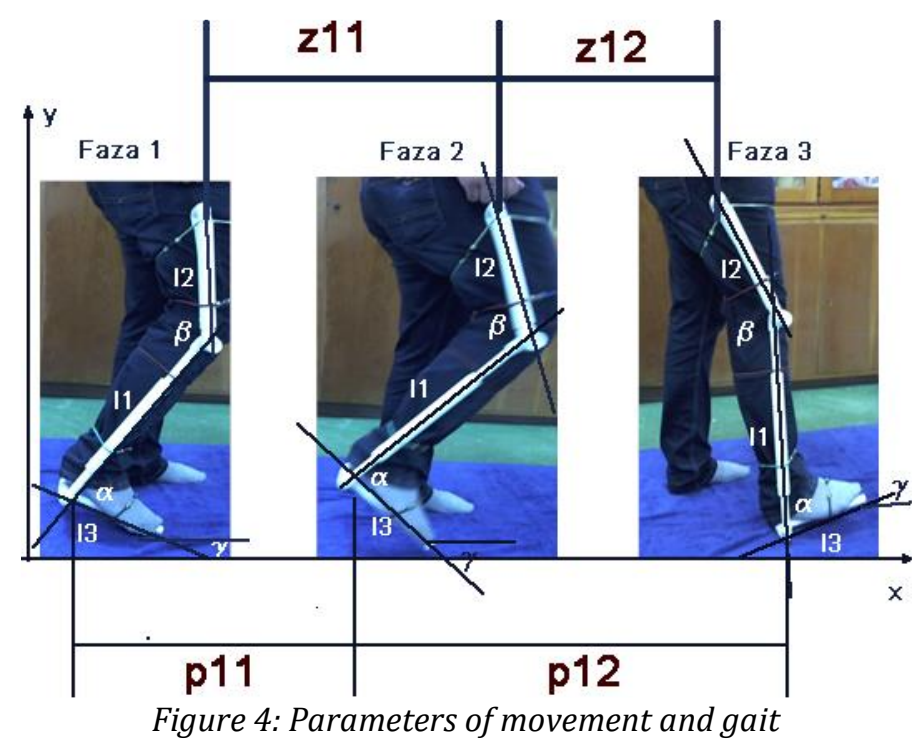

In our case, we have the advantage that we can observe the change of parameters for the entire sequence of gait.

This is possible by using a video recording system that will tailor human gait data for a specific person at a specific speed. However, it is necessary to ensure that the subject maintains the magnitude of the speed, which is not easy to achieve.

To solve this problem, a method based on the comparison of theoretical parameters with experimentally measured data in three phases was used using two control parameters z11 and z12, which will be equal to the distance traveled by a $\mathrm{z} 1$ step at the hip. These parameters can be more easily transposed to the foot by means of the parameters p11 and p12, which will be equal to the distance traveled by a step p1 at the ground level. To further reduce errors, it is necessary for the patient to maintain a maximum walking time (TF3) and to use the average values of several recorded cycles. 
In the method, the entire trajectory will be dependent on three sets of parameters: geometry, walking and control, which we present in the table.

Table 1. Model parameters and their definition

\begin{tabular}{|c|c|c|}
\hline Parameter & Definition & Obtaining \\
\hline $\mathrm{X} l 1, \mathrm{Yl1}$ & Coordinates of the ankle in the XY plane & From equation 1 \\
\hline $\mathrm{X} 12, \mathrm{y}_{12}$ & The knee coordinates in the XY plane & From equation 2 \\
\hline VXl1, Vyl1 & Ankle speeds in the XY plane & From equation 3 \\
\hline vXl2, vyl2 & Knee speeds in the XY plane & From equation 4 \\
\hline \multicolumn{3}{|c|}{ Geometric Parameters } \\
\hline$\alpha$ & The relative ankle angle of the sagittal plane & From measuring \\
\hline$\beta$ & The relative knee angle in the sagittal plane & From measuring \\
\hline$\gamma$ & $\begin{array}{l}\text { The relative angle of the virtual line between the } \\
\text { calcaneus and the distal phalange III with the } \\
\text { ground in the sagittal plane }\end{array}$ & From measuring \\
\hline $\mathrm{l}_{2}$ & Length of thigh segment & From measuring \\
\hline $\mathrm{l}_{1}$ & The length of the calf segment & From measuring \\
\hline $\mathrm{l}_{3}$ & The length of the foot segment & From measuring \\
\hline $\mathrm{l}_{31}$ & $\begin{array}{l}\text { The distance from the extreme point on the outer } \\
\text { surface of the calcaneus to the distal male (ankle) } \\
\text { measured on the X axis }\end{array}$ & From measuring \\
\hline $\mathrm{l}_{32}$ & $\begin{array}{l}\text { The distance from the distal phalange face to the } \\
\text { distal male (ankle) measured on the X axis }\end{array}$ & From measuring \\
\hline $\mathrm{l}_{33}$ & $\begin{array}{l}\text { The distance from the extreme point on the outer } \\
\text { surface of the calcaneus to the distal male (ankle) } \\
\text { measured on the Y axis }\end{array}$ & From measuring \\
\hline \multicolumn{3}{|c|}{ Gait parameters } \\
\hline $\mathrm{T}_{\mathrm{F} 3}$ & $\begin{array}{l}\text { The moment of a complete gait cycle. Coincides } \\
\text { with the final cycle. }\end{array}$ & $\begin{array}{l}\text { It is defined by the speed of travel. } \\
\text { From measuring }\end{array}$ \\
\hline $\mathrm{T}_{\mathrm{F} 1}$ & $\begin{array}{l}\text { The instant moment of start when the support on } \\
\text { two legs is made }\end{array}$ & It sets. Defines $\mathrm{T}_{\mathrm{F} 3}$ \\
\hline $\mathrm{T}_{\mathrm{F} 2}$ & $\begin{array}{l}\text { The instant moment when } x l 1 \text { and yl1 reach the } \\
\text { maximum possible }\end{array}$ & $\begin{array}{l}\text { Based on the recorded data being } \\
\text { represented by } \mathrm{T}_{\mathrm{F} 3}\end{array}$ \\
\hline $\mathrm{p}_{1}$ & The length of the step & $\begin{array}{l}\text { Based on recorded data being } \\
\text { considered constant at any speed }\end{array}$ \\
\hline$\gamma_{\mathrm{F} 1}$ & Start angle of the balance & Based on recorded data \\
\hline$\gamma_{\mathrm{F} 3}$ & Angle of laying the foot on the ground & Based on recorded data \\
\hline \multicolumn{3}{|c|}{ Control parameters } \\
\hline $\mathrm{Z} 11, \mathrm{Z} 12$ & $\begin{array}{l}\text { The distances on the x-axis from the hip to the } \\
\text { ankle of the foot }\end{array}$ & It sets \\
\hline
\end{tabular}

The first set of parameters is related to the anatomy of the patient, i.e. geometric, being defined by the length of each segment. Typically, these values are taken from catalogs with common physical properties [3], each segment being calculated based on body mass and body height.

The second set of parameters are those that are obtained based on recorded data and are considered the same at all speeds, since the length of a step is constant, so different walking speeds are needed for different gait cycles. For this reason, TF3 is defined by the speed and it can be shown that depending on the ankle and hip trajectories, all the trajectories of the other leg segments can be determined. [2].

Thus, all calculations are made for one foot and can be repeated or considered like the other foot.
Using experimental data recorded on each individual patient, it can be considered that each location of each joint can be determined to be a distinct point in the center of the joint. It is possible to interpolate these points using a function that links motion and imposing boundary conditions and geometric conditions resulting in the ankle and knee trajectories. We can consider polynomial functions that are continuous on intervals because the calculation functions must allow for each iteration to move as continuously without sudden shifts and thus their variation to be continuous and monotone. Thus, functions must be able to derive and their derivatives be continuous. Functions will be obtained by applying the recorded data to a polynomial regression. 
Images captured using a normal Logitech HD PRO Webcam C920 were first processed so that the segments to be measured are as clear as possible. For this purpose, the image has been transformed into black and white, and its contrast and brightness have changed and has been inverted. A weighting matrix was applied to analyze each bit [1] with values ranging from 0 to 1 . For each pixel, the image was normalized using an amplitude-type variable.

Then the image was divided into cells using a $128 \times 128$ cell grid, and the values were saved as values of this matrix. Single points (which have only one neighbor point of value 1 ) were eliminated and then an analysis of the identified parameters from the vector segments.

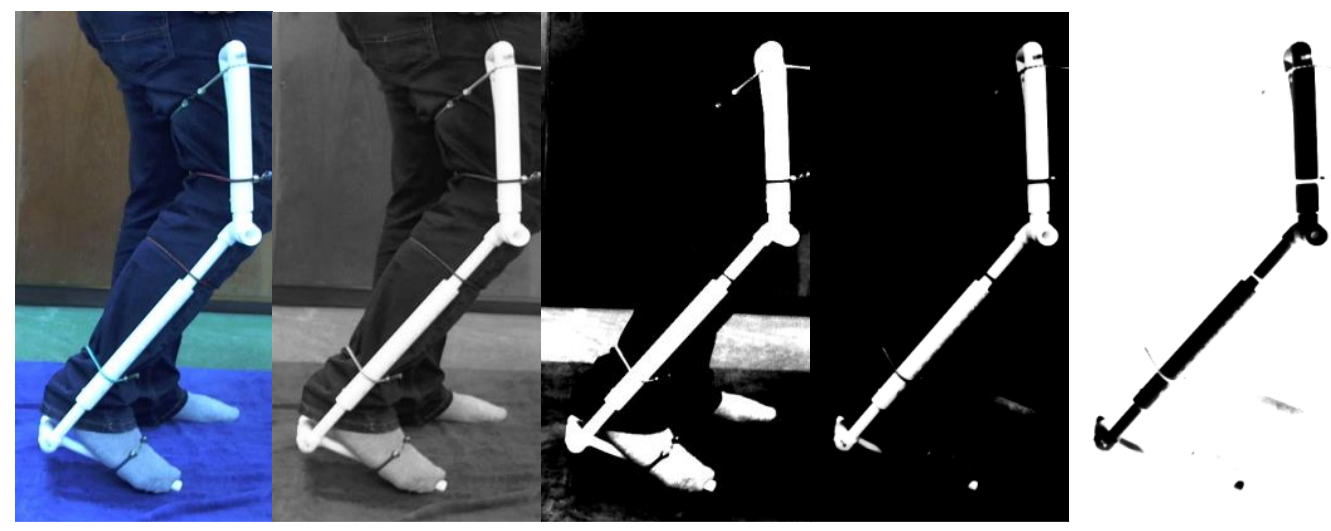

Figure 5: Image processing: A) original image; B) black and white image; C) change to + 40\% of contrast; D) modification of $-40 \%$ of brightness; E) inverted image

For each frame, a comparative scale analysis of the actual body segment dimensions was made and the measured parameters were corrected to yield results that will be presented below.

\section{Conclusion}

By exemplifying an ankle trajectory, more specifically the lateral malleolus, we can consider a polynomial function that will consider the point where the foot enters the balance (Phase 1). The function data will be of the sinusoidal type from ground contact $(0 \mathrm{~mm})$ to the maximum position $(22 \mathrm{~mm})$ and until the foot is restored to the ground
$(0 \mathrm{~mm})$ during a gait cycle of $5 \mathrm{sec}$. The segments were adjusted to $400 \mathrm{~mm}$ and $450 \mathrm{~mm}$ thigh.

The angle at the knee is between 116.4-179 degrees and the ankle between 84-90 degrees. The sequence of walking is shown in the following figures. The analysis of the image analyzed the amplitude of the kinematic parameters of the walk. We will only exemplify here the variation of the height of the height of the lateral malleolus point. It can be noticed how the subject's mobility is relatively good even though it is trying to maintain a steady displacement speed.

Parameters variation is due to errors in the measurement process, especially image vectoring.

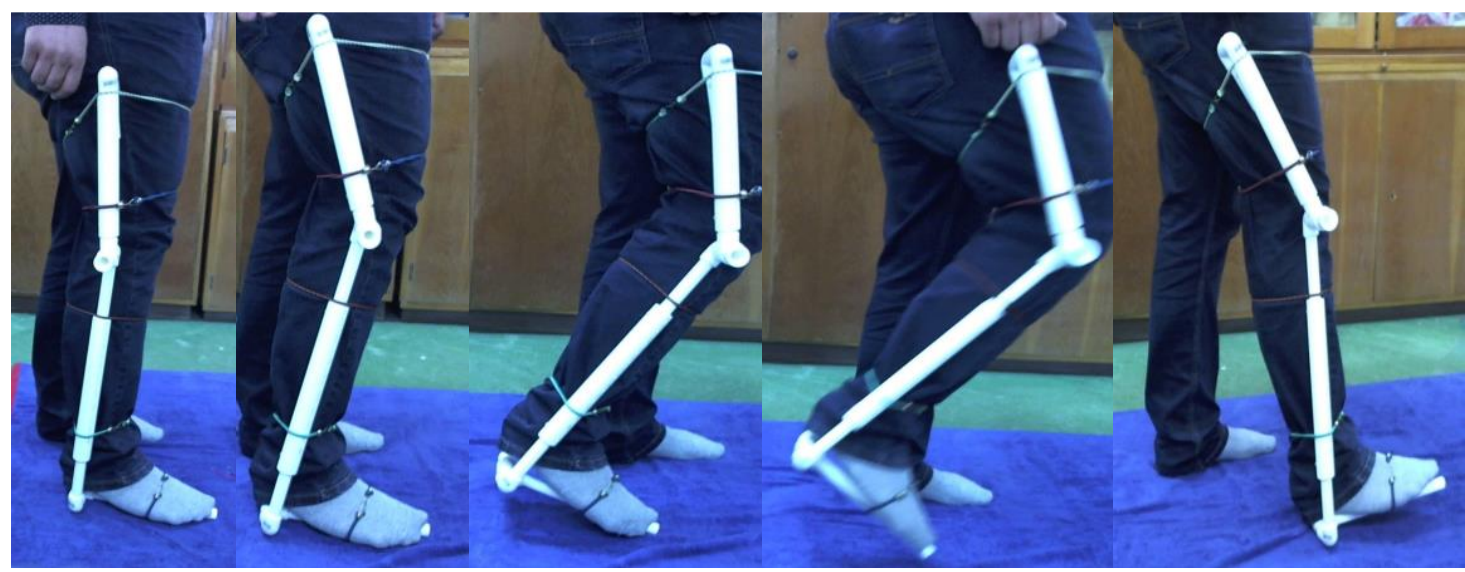

Figure 6: Gait cycle 


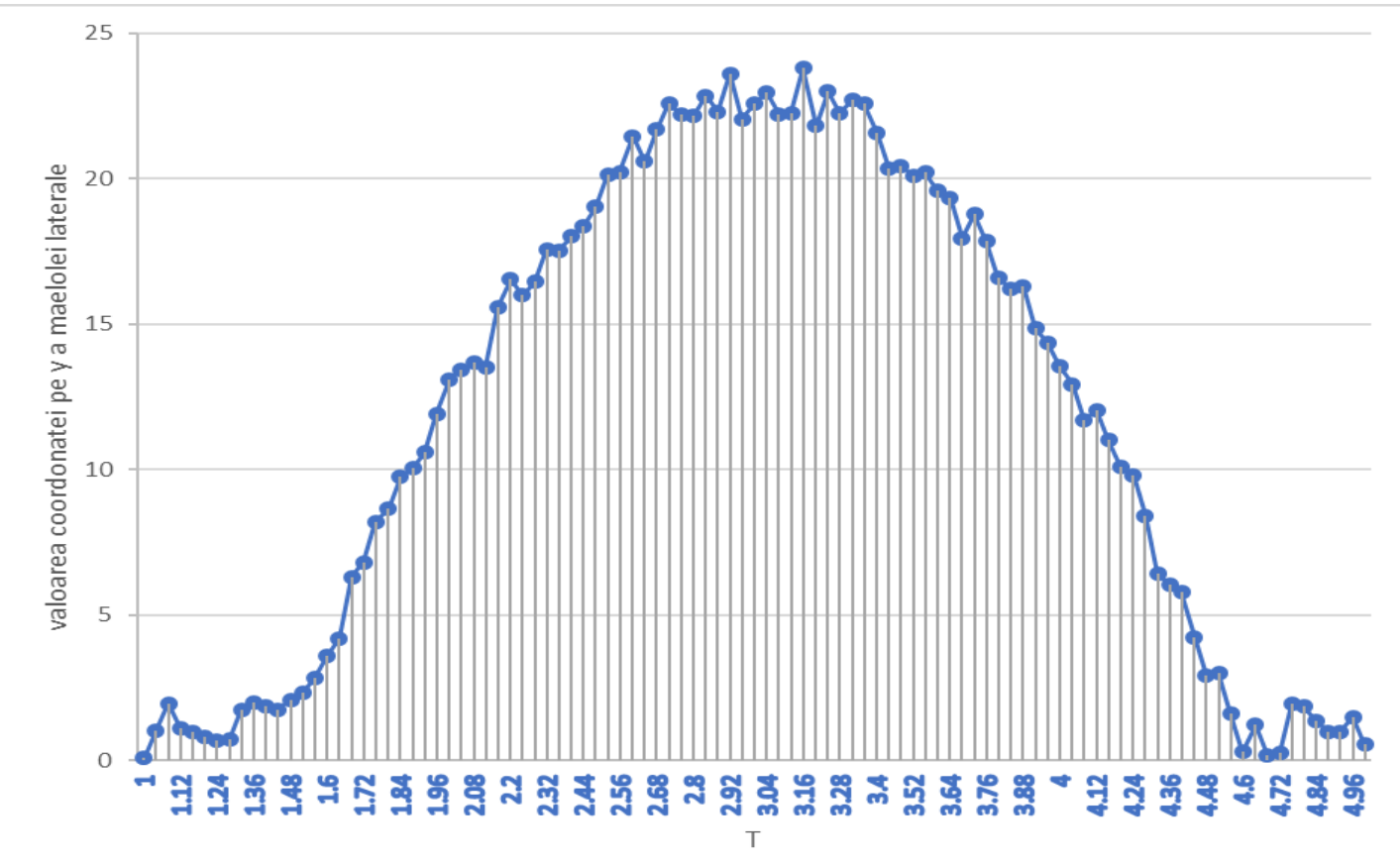

Figure 7: Ankle trajectory in the $y$ direction

In this article has been presented a simple walking assessment method for a individual inferior member, and it has been shown how to use this type of mechanical system that is useful in assessing walking for people who, for various reasons, have locomotor problems.

The method is a visual one with the advantage that it does not require special facilities and equipment based on image processing captured while driving.

The construction of the mechanical system envisaged the use of components having the lowest possible weight and low cost and was therefore chosen as polypropylene (PPR) material.

\section{References}

[1] http://www.learnopencv.com/imagerecognition-and-object-detection-part1/
[2]Huang, Q., Yokoi, K., Kajita, S., Kaneko, K., Arai, H., Koyachi, N. and Tanie, N., "Planning Gait Pattern for a Biped Robot", IEEE International Transition on Robotic and Automation, 2001 Vol. 17, No. 3, pp 280-289.

[3]Winter, D., A., "Biomechanics and motor control of human movement." 3rd Ed Wiley, 2004

[4]Kaufman, K. R., Hughes, C., Morrey, B.F., Morrey, M. and An, K.N., "Gait characteristics of patients with knee osteoarthritis" Journal of Biomechanics, July 2001, Vol. 34, Issue 7, Pages 907-915

[5]Zeni Jr., J.A., Richards, J.G. and Higginson, J.S., "Two simple methods for determining gait events during treadmill and over ground walking using kinematic data" May 2008, Vol. 27, Issue 4, Pages 710-714

[6]Dontu, O., Ciobanu, R. and Avarvarei, I. "Inertial system used to analyze the normal and pathological human gait" Romanian Review Precision Mechanics, Optics \& Mechatronics, Issue 38, 2010. 